\title{
Age-Dependent Sensitivity to the Neurotoxic Environmental Metabolite, 1,2-Diacetylbenzene
}

\author{
Ngoc Minh Hong Hoang', Sungjin Kim¹, Hai Duc Nguyen ${ }^{1}$, Minjo Kim², Jin Kim¹, Byoung-Chul Kim², \\ Daeui Park ${ }^{3}$, Sujun Lee ${ }^{4}$, Byung Pal Yu ${ }^{5}$, Hae Young Chung ${ }^{2, *}$ and Min-Sun Kim ${ }^{1, *}$ \\ ${ }^{1}$ Department of Pharmacy, College of Pharmacy and Research Institute of Life and Pharmaceutical Sciences, Sunchon National \\ University, Suncheon 57922, \\ ${ }^{2}$ Molecular Inflammation Research Center for Aging Intervention (MRCA), Department of Pharmacy, College of Pharmacy, Pusan \\ National University, Busan 46241 , \\ ${ }^{3}$ Systems Toxicology Research Center, Korea Institute of Toxicology, Daejeon 34114, \\ ${ }^{4}$ Department of Pharmacology, College of Medicine, Inje University, Busan 47392, Republic of Korea \\ ${ }^{5}$ Department of Physiology, The University of Texas Health Science Center at San Antonio, San Antonio, TX 78229, USA
}

\begin{abstract}
1,2-Diacetylbenzene (DAB) is a metabolite of 1,2-diethylbenzene, which is commonly used in the manufacture of plastics and gasoline. We examined the neurotoxic effects of DAB in young and old rats, particularly its effects on hippocampus. Previously, we reported DAB impairs hippocampal neurogenesis but that the underlying mechanism remained unclear. In this study, we evaluate the toxicities exhibited by DAB in the hippocampi of 6-month-old (young) and 20-month-old (old) male SD rats by treating animals intraperitoneally with DAB at $3 \mathrm{mg} / \mathrm{kg} /$ day for 1 week. Hippocampal areas were dissected from brains and RNA was extracted and subjected to RNA-seq analysis. RNA results showed animals exhibited age-dependent sensitivity to the neurotoxic effects of DAB. We observed that inflammatory pathways were up-regulated in old rats but that metabolism- and detoxification-related pathways were up-regulated in young rats. This result in old rats, especially upregulation of the TREM1 signaling pathway (an inflammatory response involved in Alzheimer's disease (AD)) was confirmed by RT-PCR. Our study results provide a better understanding of age-dependent responses to DAB and new insight into the association between DAB and AD.
\end{abstract}

Key Words: Neurotoxicity, 1,2-Diacetylbenzene, RNA-seq, DEB, Aging

\section{INTRODUCTION}

Organic solvents are commonly used in industry (Dick, 2006). 1,2-Diethyl benzene (DEB) is a component of gasoline and jet fuel (Thrall et al., 2007) and can be easily absorbed through skin or by inhalation (Kim et al., 2007). 1,2-Diacetylbenzene (DAB) is a neurotoxic metabolite of DEB (Cavender, 1994) and can induce central and peripheral neuropathies that result in motor neuronal deficits (Sabri et al., 2007) and impair hippocampal neurogenesis (Kim et al., 2011). In addition, DAB increases oxidative stress, activates microglia, and causes memory deficits via tau hyperphosphorylation (Kang et al., 2017). Furthermore, it has been well established that oxidative stress is associated with age-related degenerative conditions and plays an important role in the inflammatory processes involved in diseases, including Alzheimer's disease (Liguori et al., 2018; Sarkar et al., 2019). The hippocampus is a major contributor to learning and memory and is disrupted in neurodegenerative diseases, especially in Alzheimer's disease (AD) (Anand and Dhikav, 2012).

Environmental toxic stress can adversely affect health and lead to lifelong problems in learning, behavior, and physical and mental health (Anand and Dhikav, 2012; Franke, 2014). Furthermore, the proportion of elderly people is increasing worldwide, and it has been estimated that the number of people aged $\geq 60$ years will reach over 2 billion in 2050 (Stambler et al., 2018). In the toxicology field, research efforts have focused on young adults rather than the elderly, and older

\section{Open Access https://doi.org/10.4062/biomolther.2020.208}

This is an Open Access article distributed under the terms of the Creative Commons Attribution Non-Commercial License (http://creativecommons.org/licenses/by-nc/4.0/) which permits unrestricted non-commercial use, distribution, and reproduction in any medium, provided the original work is properly cited.
Received Nov 16, 2020 Revised Jan 13, 2021 Accepted Feb 10, 2021 Published Online Apr 6, 2021

\section{*Corresponding Authors}

E-mail: minsun@scnu.ac.kr (Kim MS), hyjung@pusan.ac.kr (Chung HY) Tel: +82-61-750-3756 (Kim MS), +82-51-510-2814 (Chung HY) Fax: +82-61-750-3708 (Kim MS), +82-51-518-2821 (Chung HY) 
people have different biologic responses to xenobiotics due in part to reduced liver and kidney functions (Kim et al., 2015; Denic et al., 2016). Therefore, we considered study of toxic responses in older people was warranted to address concerns related to societal aging (Geller and Zenick, 2005).

To investigate the different responses between young and old condition, we chose 6-month-old and 20-month-old rats to represent the two groups. Laboratory rats have an average life span of about 2-3.5 years and reach their sexual maturity at about six weeks of age (Sengupta, 2013). Six months and 20 months are equal to 18 years and around 50 years old in humans, respectively. In this study, we evaluated the neurotoxic effects of DAB in 6-month-old (young) and 20-month-old (old) male SD rats by genome-scale transcriptome analysis using RNA-Seq (Ji and Sadreyev, 2018). Young and old rats were treated with $3 \mathrm{mg} / \mathrm{kg} /$ day intraperitoneally for 1 week with the aim of identifying the different mechanisms responsible for the neurotoxicity of DAB in old and young subjects.

\section{MATERIALS AND METHODS}

\section{Animal treatment and sample preparation}

Young (6-month-old) and old (20-month-old) specific pathogenfree male Sprague-Dawley rats were obtained from Samtako, Inc (Osan, Korea). Temperature and humidity were maintained at $23-25^{\circ} \mathrm{C}$ and $45-55 \%$, respectively, and animals were maintained under a $12 \mathrm{~h}$ light/12 $\mathrm{h}$ dark diurnal cycle. Food and water were provided ad libitum. Young and old rats were assigned to control and DAB-treated groups at 3 rats per group. Animals were acclimatized for 1 -week before experiments and then treated with saline (controls) or DAB at $3 \mathrm{mg} / \mathrm{kg} / \mathrm{day}$ in saline i.p. for 1 week. Experiments were performed in accordance with the animal care guidelines issued by the Pusan National University Animal Care Committee (Pusan National University, Busan, Korea). After treatment, rats were sacrificed by decapitation, and hippocampi destined for RNA extraction were quickly dissected, immediately frozen in liquid nitrogen, and stored at $-80^{\circ} \mathrm{C}$ for RNA extraction.

\section{Isolation of total RNA from hippocampus}

Total hippocampal RNA was purified using TRIzol reagent
(Invitrogen, Carlsbad, CA, USA), according to the manufacturer's instructions. Homogenates of hippocampal samples in TRIzol reagent ( $1 \mathrm{~mL}$ per $50 \mathrm{mg}$ tissue) were incubated for 5 minutes at room temperature (RT), $0.2 \mathrm{~mL}$ chloroform was added, incubated for 2 min at RT, and centrifuged at 12,000 $\mathrm{g}$ for $10 \mathrm{~min}$ at $4^{\circ} \mathrm{C}$. The aqueous phase was transferred to a fresh tube, $0.5 \mathrm{~mL}$ of isopropyl alcohol (Sigma Aldrich, MO, USA) was added per $1 \mathrm{~mL}$ of TRIzol reagent, incubated for 10 minutes at RT, and centrifuged at $12,000 \mathrm{~g}$ for 10 minutes at $4^{\circ} \mathrm{C}$. RNA pellets were washed by adding $0.5 \mathrm{~mL}$ of $75 \%$ ethanol and centrifuging at $8,000 \mathrm{~g}$ for 5 minutes at $4^{\circ} \mathrm{C}$, supernatants were carefully discarded, washed with ethanol, and air-dried for 5 minutes. RNAs were then dissolved in water containing DEPC (diethyl pyrocarbonate 1\%, v/v), incubated for $10-15$ minutes at $56^{\circ} \mathrm{C}$, and checked for quality by measuring optical density ratios: OD ${ }_{260 / 280}$ and $O D_{260 / 230 .}$

\section{RNA sequencing}

An RNA sequencing library was generated using the TruSeq RNA sample preparation kit (Illumina, San Diego, CA, USA). Briefly, mRNA was separated from total RNA using Oligo (dT) beads and chemically fragmented. After double-strand cDNA synthesis of fragmented mRNA, end-repair, adenylation of 3'ends, and sequencing adapter ligation, DNA purification with magnetic beads and PCR amplification were carried out. Finally, amplified libraries were purified, quantified, and subjected to template preparation. The HiSeq2500 platform was used to generate 101-bp paired-end sequencing reads (Illumina).

\section{Genome mapping and identification of differentially expressed genes}

All 101-bp paired-end sequence reads were preprocessed by eliminating adaptor sequences and low quality reads using BBDuk in BBMap version 34.90 (https://jgi.doe.gov/data-andtools/bbtools/). Cleaned sequence reads were mapped to the rat transcriptome (Ensembl Rnor_6.0) using Bowtie2 version 2.2.5 (Langmead and Salzberg, 2012) and RSEM package version 1.2.21 ( $\mathrm{Li}$ and Dewey, 2011). Finally, differentially expressed genes (DEGs) were identified using EBseq in the $R$ package (Leng et al., 2013). Significantly different mRNA expression was defined as a fold change threshold of 2 and a posterior probability of a differential expression (PPDE)

Table 1. Primer sequences for RT-PCR analysis $\left(5^{\prime} \rightarrow 3^{\prime}\right)$

\begin{tabular}{lll}
\hline \multirow{2}{*}{ Primers } & \multicolumn{1}{c}{ Forward } & Sequence $\left(5^{\prime} \rightarrow 3^{\prime}\right)$ \\
\cline { 2 - 3 } & \multicolumn{1}{c}{ Reverse } \\
\hline IL-1 $\beta$ & GACTTCACCATGGAACCCGT & GGAGACTGCCCATTCTGCAC \\
iNOS & GTTCCTCAGGCTTGGGTCTT & TGGGGGACACAGTAATGGC \\
DAP12 & ACAGGCCCAGACTGACAATTA & GGGCATAGGGTGGGTTCATC \\
NF-kB & GGTGTGTCCGTTCCTGACAT & CCTCTTTCCTGAGGGTGCAG \\
Casp1 & TTCAACATGGCAGACGACGA & CCATCTGTTGACAGTGGTATATCTG \\
TLR4 & GACCGAGTGGTTCCCTCAAG & GACGTGTACGAGTGGGTTT \\
C3a & CGCTTTCAGCTTTGCCTTCA & CTCCAGAAGATGTGCCTCCC \\
C5a & GGTCAGTACACCGACAAGGG & TCTCTTCTGTGCTGCTCACG \\
GAPDH & TGCTGTTATGATGGAGCCCG & CTGATGCAGTGTGGGCCTAT \\
\hline
\end{tabular}

TREM1, Triggering Receptors expressed on myeloid Cells 1; TLR4, Toll-like receptor 4; C3a, C5a, complement components C3a, C5a; DAP-12, 12-kDa DNAX activating protein; Casp1, caspase 1; IL-1 $\beta$, interleukin 1 beta; NF-kB, nuclear factor kappa-light-chain-enhancer of activated B cells; iNOS, inducible nitric oxide synthase; GAPDH, glyceraldehyde-3-phosphate dehydrogenase. 
threshold of 0.95 .

\section{Gene set enrichment testing and functional categorization}

To characterize biological pathways associated with DAB, DEGs were analyzed using Ingenuity Pathways Analysis software (IPA; Ingenuity Systems, Redwood City, CA, USA) for biological functions and canonical pathways.

\section{Protein network predictions}

Protein-protein interaction (PPI) networks were generated by retrieving relatively high confidence protein interactions (confidence score cut-off of 0.7) from the STRING database (Szklarczyk et al., 2015). The resulting network was visualized using Cytoscape and network metrics (degree and betweenness centrality) were calculated using Network Analyzer v.2.7 in Cytoscape plugin (Kohl et al., 2011) (Supplementary Table 1, 2).

\section{Quantitative RT-PCR}

Real-time PCR was performed on cDNA samples by using $\mathrm{iQ}^{\mathrm{TM}}$ SYBR Green Supermix and the MiniOpticon Real-time PCR system (Bio-Rad, Hercules, CA, USA). Primers were supplied by Bioneer (Daejeon, Korea) and are detailed in Table 1. The protocols used were as follows: denaturation $\left(95^{\circ} \mathrm{C}\right.$ for $10 \mathrm{~min}), 44$ amplification cycles $\left(94^{\circ} \mathrm{C}\right.$ for $20 \mathrm{~s}, 60^{\circ} \mathrm{C}$ for 20 $\mathrm{s}, 72^{\circ} \mathrm{C}$ for $20 \mathrm{~s}$ ), melting (from $55^{\circ} \mathrm{C}$ to $95^{\circ} \mathrm{C}, 5^{\circ} \mathrm{C}$ increment for $5 \mathrm{~s}$ ) and data acquisition. The analysis was conducted using sequence detection software (Bio-Rad CFX Manager 2.1, Bio-Rad) supplied with the instrument. For each sample, delta delta Threshold Cycle (ddCT) (crossing point) values were calculated by subtracting the cycle threshold (Ct) of GAPDH genes from that of the target gene. Gene expressions were derived using the $2^{- \text {ddct }}$ equation, and changes in gene expressions are expressed relative to basal levels.

\section{Statistical analysis}

The significant differences between the control and DABtreated groups were assessed using Student's $t$-test. All analyses were done in GraphPad Prism 8.1 (GraphPad Software, Inc., San Diego, CA, USA). Differences between groups were considered statistically significant for $p<0.05$.

\section{RESULTS}

\section{Detection of DAB-induced changes in gene expressions in young and old rats}

The genome-wide mRNA expression profiles of young and old rats treated with vehicle or DAB were compared using EBseq results. $D A B$ treatment resulted in the up- and down-regulations of 490 and 113 genes, respectively, in old rats when an expressional change cut-off of $\geq 2$-fold was applied, and in the up- and down-regulations of 82 and 276 genes, respectively, in young rats (Fig. 1A). Only 41 of these DEGs overlapped in old and young rats (Fig. 1B). In which, 12 genes were upregulated and 7 genes were downregulated in both old and young rats (Table 2). To view overall gene expression changes, the distribution of mRNA expression of each group was shown in Fig. 1C. Overall, DAB-induced responses in rats varied by age; old rats were more affected than young rats in terms of both gene expression levels and numbers.

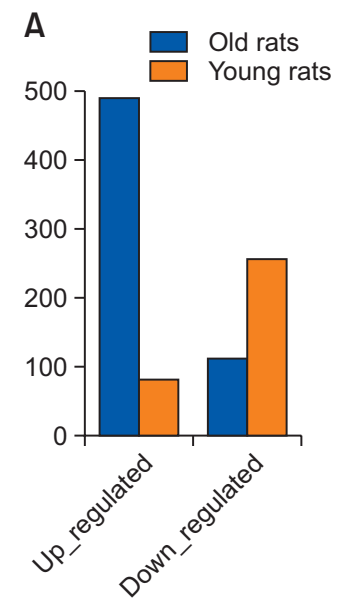

B
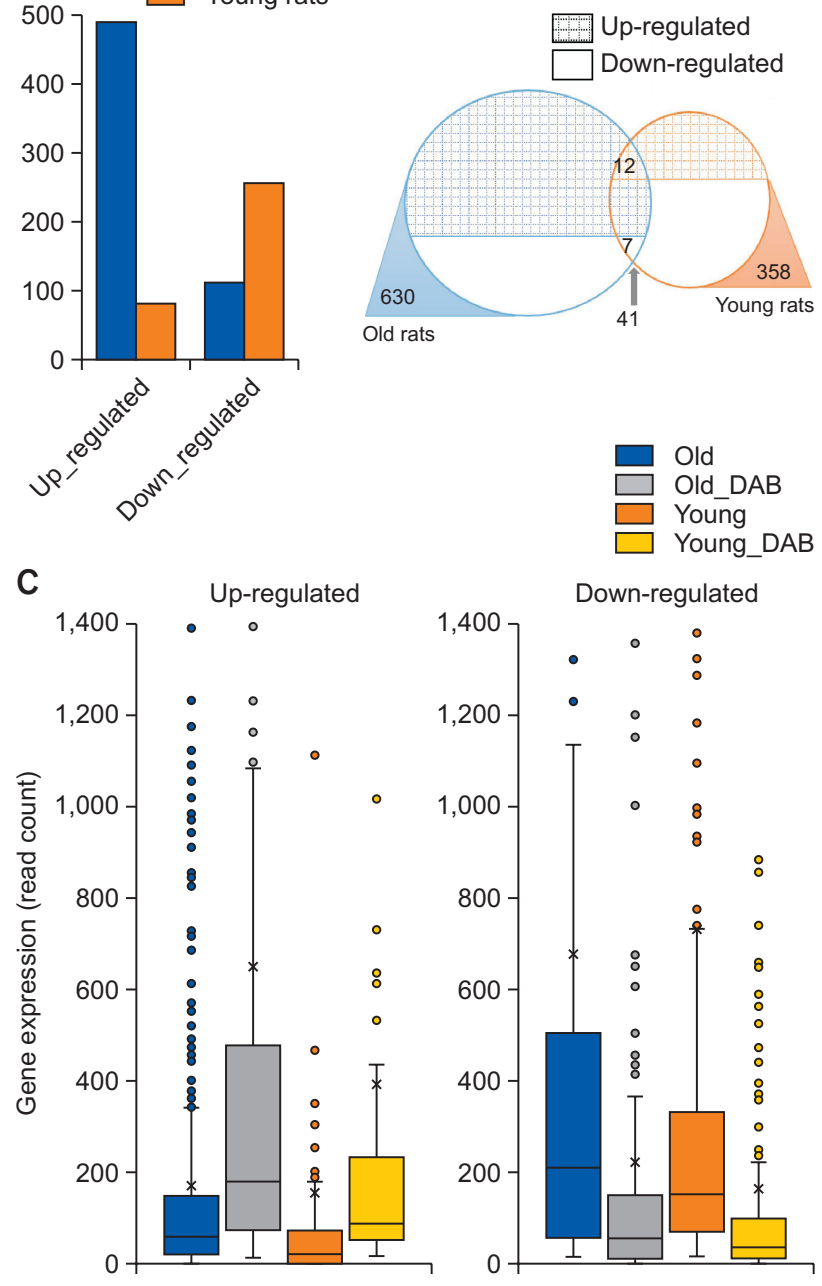

Fig. 1. Genes differentially expressed in old and young rats. Differentially expressed genes were filtered at $p<0.01$ and fold change $\geq 2$. (A) 490 up- and 113 down-regulated genes were identified in old rats and 82 up- and 276 down-regulated genes in young rats. (B) Number of genes overlapped between the two groups. (C) Distribution of mRNA expressions in the study groups.

\section{Gene set enrichment analysis of up- and down-regulated genes}

To classify DEGs, we analyzed the biological pathways of genes differentially expressed after DAB treatment using IPA software. In old rats, the genes of 111 pathways were significantly upregulated, while the genes of 4 pathways were significantly downregulated (filtered at $p<0.01$; Table $3 A$ ). In young rats, the genes of 25 pathways were upregulated and the genes of 6 pathways were downregulated (filtered at $p<$ 0.01 ; Table 3B).

In old rats, immune-related pathways such as dendritic cell maturation, the Triggering Receptor Expressed on Myeloid Cells (TREM1) pathway, T and B cell signaling pathways, complement system, and cytokine signaling were upregulated. TREM1 is known to play important roles in innate immune responses, such as in the activation of inflammatory responses, and contributes to septic shock response to microbial-mediat- 
Table 2. Genes that are either up-regulated or down-regulated in both young and old rats

\begin{tabular}{|c|c|c|c|c|}
\hline & \multirow{2}{*}{ Gene } & \multirow{2}{*}{ Description } & \multicolumn{2}{|c|}{ Fold change } \\
\hline & & & Young & Old \\
\hline \multirow[t]{12}{*}{ Up } & LOC100911186 & Enoyl-CoA hydratase, mitochondrial-like & 22898.08 & 4.19 \\
\hline & PNPLA2 & Patatin-like phospholipase domain containing 2 & 16619.56 & 17499.59 \\
\hline & FAM50A & Family with sequence similarity 50 , member $A$ & 13310.83 & 6.80 \\
\hline & SPATC1L & Spermatogenesis and centriole associated 1-like & 5155.26 & 7150.47 \\
\hline & INTS1 & Integrator complex subunit 1 & 2739.23 & 3.86 \\
\hline & AFM & Afamin & 6.54 & 10.15 \\
\hline & TUBA1C & Tubulin, alpha $1 \mathrm{C}$ & 3.85 & 3.63 \\
\hline & KNG1L1 & Kininogen 1-like 1 & 3.84 & 2.34 \\
\hline & ALB & Albumin & 3.52 & 2.10 \\
\hline & MFF & Mitochondrial fission factor & 2.68 & 4.50 \\
\hline & AABR07065493.1 & Uncharacterized protein & 2.62 & 4393.26 \\
\hline & LOC100911833 & Murinoglobulin-2-like & 2.46 & 2.23 \\
\hline \multirow[t]{7}{*}{ Down } & UBE2B & Ubiquitin-conjugating enzyme E2B & -2743.23 & -3168.48 \\
\hline & VKORC1L1 & Vitamin $\mathrm{K}$ epoxide reductase complex, subunit 1-like 1 & -1884.63 & -1909.02 \\
\hline & LRRC23 & Leucine rich repeat containing 23 & -1366.03 & -1292.31 \\
\hline & AC106663.1 & & -343.69 & -2.49 \\
\hline & AABR07059639.1 & & -6.22 & -2.15 \\
\hline & GPR88 & G-protein coupled receptor 88 & -2.79 & -2.12 \\
\hline & ANKRA2 & Ankyrin repeat, family A (RFXANK-like), 2 & -2.45 & -2.02 \\
\hline
\end{tabular}

ed infections (Colonna and Facchetti, 2003).

In young rats, up-regulated pathways mainly were related to metabolism and detoxication e.g., activation of farnesoid $X$ receptor/ retinoid $X$ receptor (FXR/RXR), liver $X$ receptor/retinoid $X$ receptor ( $L X R / R X R)$, chemical degradation pathways, and xenobiotic metabolism signaling pathways. Some inflammation and oxidative stress-related pathways, including acute phase response signaling, IL-12 signaling, and production in macrophages, production of nitric oxide and reactive oxygen species, were upregulated both in young and old rats.

\section{Protein network prediction and inflammation-related proteins}

Protein networks were constructed for the 584 up- and 862 down-regulated proteins in old rats (Fig. 2), and 326 up- and 121 down-regulated proteins in young rats (Fig. 3). Node size indicates betweenness centrality.

Inflammation-related genes were induced by DAB in old rats more than young rats. Protein network in old rats shows that DAB mainly affected inflammation-associated proteins such as tumor necrosis factor (TNF), spleen tyrosine kinase (SYK), tyrosine-protein kinase (LYN), tyrosine-protein phosphatase non-receptor type 6 (PTPN6), and Ras-related C3 botulinum toxin substrate 2 (RAC2). Whereas DAB mainly affected xenobiotic metabolism-related proteins such as CYP4, CYP1, and UDP-glucuronosyl transferases (UGT) in young rats.

\section{RT-PCR results: Inflammation and neurotoxicity in old rats}

The TREM signaling pathway was upregulated in old rats. This pathway is responsible for amplifying or attenuating TLRinduced signals, and thus, plays important roles in inflammatory response (Fig. 4).

To confirm the DEGs identified by RNA-seq, we selected 9 genes involved in TREM signaling based on pathway results (Fig. 2A) and examined their relative gene expressions by RT-PCR. Fold change values are shown in Fig. 5 . The re- sult obtained showed that the expressions of all 9 genes were increased by DAB in both young and old rats. However, significant fold increases were observed in old rats, which concurred with RNA-seq results, whereas inconsistencies were observed RNA-seq and RT-PCR results in young rats. A comparison of relative fold changes between RNA-seq and RTPCR results with respect to TREM signaling genes is shown in Fig. 6. DEG results showed 11 TREM1 signaling genes (TREML1, TLR-4, C3, C5aR1, C5aR2, TYROBP (DAP-12),

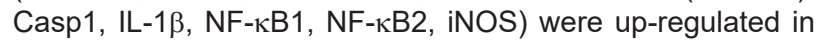
old rats, whereas in young rats only C3, IL-1 $\beta$, and iNOS were up-regulated, TREML1, TLR-4, Casp1, NF-кB2 were downregulated, and the expressions of C5aR1, C5aR2, DAP-12 were not presented.

\section{DISCUSSION}

Organic solvents can have neurological impacts after short(Wood and Liossi, 2005) or long-term exposure (Berr et al., 2010), and many studies have reported on the susceptibilities of men, women, pregnant women, and children to organic solvent exposure (Costet et al., 2018; Oliveira et al., 2018). However, the susceptibility of older people to xenobiotics, especially organic solvents, has not been as seriously addressed.

Our transcriptome analysis using RNA-seq showed that DAB had different effects on young and old rats. In particular, inflammation and ROS-related pathways were highly up-regulated in old rats. Pathway analysis showed gene expression changes well-matched those caused by the bacterial endotoxin lipopolysaccharide (LPS). LPS is a typical inflammatory model, microglial regulation and NF-kB involvement are components of LPS-induced inflammation(Dresselhaus and Meffert, 2019). Furthermore, the neurotoxic effects of LPS have been reported to be due to its inflammatory effects on nigral dopaminergic neurons (Dutta et al., 2008). 
Table 3. Pathways significantly influenced by DAB (filtered at $p<0.01$ ). 111 and 4 pathways were up- or down-regulated in old rats $(A)$, while 25 and 6 pathways were up- or down-regulated young rats $(B)$ A

\begin{tabular}{|c|c|c|c|c|}
\hline & Ingenuity canonical pathways & $p$-value & Ratio & Molecules \\
\hline \multirow[t]{9}{*}{ Up } & Dendritic cell maturation & $5.01 \mathrm{E}-21$ & $1.73 \mathrm{E}-01$ & $\begin{array}{l}\text { PIK3R6, MYD88, HLA-DQA1, FCGR1A, PIK3R5, TLR9, } \\
\text { FCER1G, CD40,TLR4, LOC100909593/RT1-DMa, IRF8, } \\
\text { LOC100909630/RT1-DMb, STAT1, TYROBP, HLA-DRB5, } \\
\text { TLR2, CD86, HLA-DOA, IL1B,HLA-DQB1, TNFRSF1B, CO- } \\
\text { L1A2, B2M, HLA-DRA, FCGR2A, PIK3CG, HLA-A, FCGR2B, } \\
\text { TLR3, IL1A, FCGR3A/FCGR3B }\end{array}$ \\
\hline & TREM1 signaling & $7.94 \mathrm{E}-18$ & 2.67E-01 & $\begin{array}{l}\text { TLR7, MYD88, IL1B, CASP1, TLR9, CD40, Naip1 (includes } \\
\text { others), TLR4, LAT2, FCGR2B, TLR3, NLRP3, TYROBP, CCL2, } \\
\text { NIrp1a, TLR10, TLR2, CD86, NLRC4, CIITA }\end{array}$ \\
\hline & $\begin{array}{l}\text { Role of pattern recognition } \\
\text { receptors in recognition of } \\
\text { bacteria and viruses }\end{array}$ & $3.16 \mathrm{E}-15$ & 1.73E-01 & $\begin{array}{l}\text { PIK3R6, TLR7, C3, MYD88, IL1B, C1QB, OAS2, PIK3R5, } \\
\text { CASP1, TLR9, C3AR1, C1QA, PIK3CG, TLR4, C1QC, SYK, } \\
\text { TLR3, C5AR1, NLRP3, IL1A, TLR2, NLRC4 }\end{array}$ \\
\hline & Complement system & $2.51 \mathrm{E}-12$ & 3.24E-01 & $\begin{array}{l}\text { ITGAM, C1QC, C4A/C4B, C2,C3, C5AR1, C1QB, ITGB2, SERP- } \\
\text { ING1, CFH, C3AR1, C1QA }\end{array}$ \\
\hline & $\mathrm{NF}-\kappa \mathrm{B}$ signaling & $8.70 \mathrm{E}-10$ & 1.1E-01 & $\begin{array}{l}\text { TNFSF13B, PIK3R6, TLR7, MYD88, IL1B, TNFRSF1B, PIK3R5, } \\
\text { TLR9, FCER1G, CD40, PIK3CG, TLR4, CARD11, TLR3, } \\
\text { MAP3K1, IL1A, TLR10, TLR2, CASP8 }\end{array}$ \\
\hline & Toll-like receptor signaling & $1.35 \mathrm{E}-06$ & $1.35 \mathrm{E}-01$ & TLR4, TLR7, MYD88, IL1B, TLR3, MAP3K1, IL1A, TLR9, TLR10, TLR2 \\
\hline & $\begin{array}{l}\text { Granulocyte adhesion and } \\
\text { diapedesis }\end{array}$ & 7.70E-06 & 7.91E-02 & $\begin{array}{l}\text { IL1RL1, ITGAM, ITGAL, IL1B, CXCL10, TNFRSF1B, } \\
\text { CSF3R,ITGB2, CXCL17, C5AR1, CXCL13, CCL2, IL1A, } \\
\text { CXCL16, ADCY4, MYD88, ADCY7, MAP3K1, IL1A, GNA15 }\end{array}$ \\
\hline & IL-1 signaling & 7.76E-03 & $6.59 \mathrm{E}-02$ & ADCY4, MYD88, ADCY7, MAP3K1, IL1A, GNA15 \\
\hline & $\begin{array}{l}\text { iNOS signaling } \\
\text { etc. }\end{array}$ & $9.55 \mathrm{E}-03$ & 9.09E-02 & TLR4, IRF1, MYD88, STAT1 \\
\hline \multirow[t]{4}{*}{ Down } & IL-17 signaling & 2.14E-03 & 4.17E-02 & PTGS2, TIMP1, MUC5B \\
\hline & TGF- $\beta$ signaling & 3.71E-03 & 3.45E-02 & INHBA, ACVR1C, PMEPA1 \\
\hline & Anandamide degradation & $7.08 \mathrm{E}-03$ & $5 \mathrm{E}-01$ & FAAH \\
\hline & Inhibition of matrix metalloproteases & 8.51E-03 & $5.13 \mathrm{E}-02$ & MMP9, TIMP1 \\
\hline
\end{tabular}

B

\begin{tabular}{|c|c|c|c|c|}
\hline & Ingenuity canonical pathways & $p$-value & Ratio & Molecules \\
\hline \multirow[t]{10}{*}{ Up } & FXR/RXR activation & $1.32 \mathrm{E}-10$ & 7.09E-02 & GC, APOB, APOA1, FETUB, SERPINA1, HPX, SULT2A1, AMBP, ALB \\
\hline & LXR/RXR activation & 2.63E-09 & 6.61E-02 & GC, APOB, APOA1, SERPINA1, HPX, AMBP, ECHS1, ALB \\
\hline & Acute phase response signaling & $6.76 \mathrm{E}-07$ & 4.14E-02 & FGB, APOA1, SERPINA1, HPX, AMBP, ALB, FGG \\
\hline & Nicotine degradation III & 2.00E-05 & 7.41E-02 & UGT2B7, CYP3A5, CYP2C19, CYP1B1 \\
\hline & Melatonin degradation I & 2.51E-05 & 7.02E-02 & UGT2B7, CYP3A5, CYP2C19, CYP1B1 \\
\hline & Superpathway of melatonin degradation & 2.51E-05 & $6.45 \mathrm{E}-02$ & UGT2B7, CYP3A5, CYP2C19, CYP1B1 \\
\hline & Nicotine degradation II & 3.72E-05 & $6.35 \mathrm{E}-02$ & UGT2B7, CYP3A5, CYP2C19, CYP1B1 \\
\hline & Bupropion degradation & 5.50E-05 & $1.2 \mathrm{E}-01$ & CYP3A5, CYP2C19, CYP1B1 \\
\hline & $\begin{array}{l}\text { Acetone degradation I } \\
\text { (to methylglyoxal) }\end{array}$ & $6.92 \mathrm{E}-05$ & $1.11 \mathrm{E}-01$ & CYP3A5, CYP2C19, CYP1B1 \\
\hline & etc. & & & \\
\hline \multirow[t]{6}{*}{ Down } & $\begin{array}{l}\text { Hepatic fibrosis / hepatic stellate } \\
\text { cell activation }\end{array}$ & 8.13E-04 & 4.37E-02 & MYH3, COL8A2, FGF1, MMP2, COL28A1, TGFBR1, COL8A1, IGF2 \\
\hline & Leukocyte extravasation signaling & $5.50 \mathrm{E}-03$ & $3.54 \mathrm{E}-02$ & $\begin{array}{l}\text { ARHGAP6, MMP2, CLDN3, ARHGAP8/PRR5-ARHGAP8, } \\
\text { MAPK13, CLDN2, MMP14 }\end{array}$ \\
\hline & G-protein coupled receptor signaling & 5.46E-03 & $3.12 \mathrm{E}-02$ & DRD1, DRD2, CAMK2D, HTR2C, ADRA2A, GRM8, RGS4, NPR3 \\
\hline & Inhibition of matrix metalloproteases & 8.32E-03 & 7.69E-02 & MMP2, THBS2, MMP14 \\
\hline & cAMP-mediated signaling & 9.33E-03 & $3.2 \mathrm{E}-02$ & DRD1, DRD2, CAMK2D, ADRA2A, GRM8, RGS4, NPR3 \\
\hline & Gai signaling & 9.33E-03 & 4.17E-02 & DRD2, ADRA2A, GRM8, RGS4, NPR3 \\
\hline
\end{tabular}

Enrichment ratio is calculated by the number of genes from the list that maps to the pathway divides to the total number of genes that map to the same pathway. 

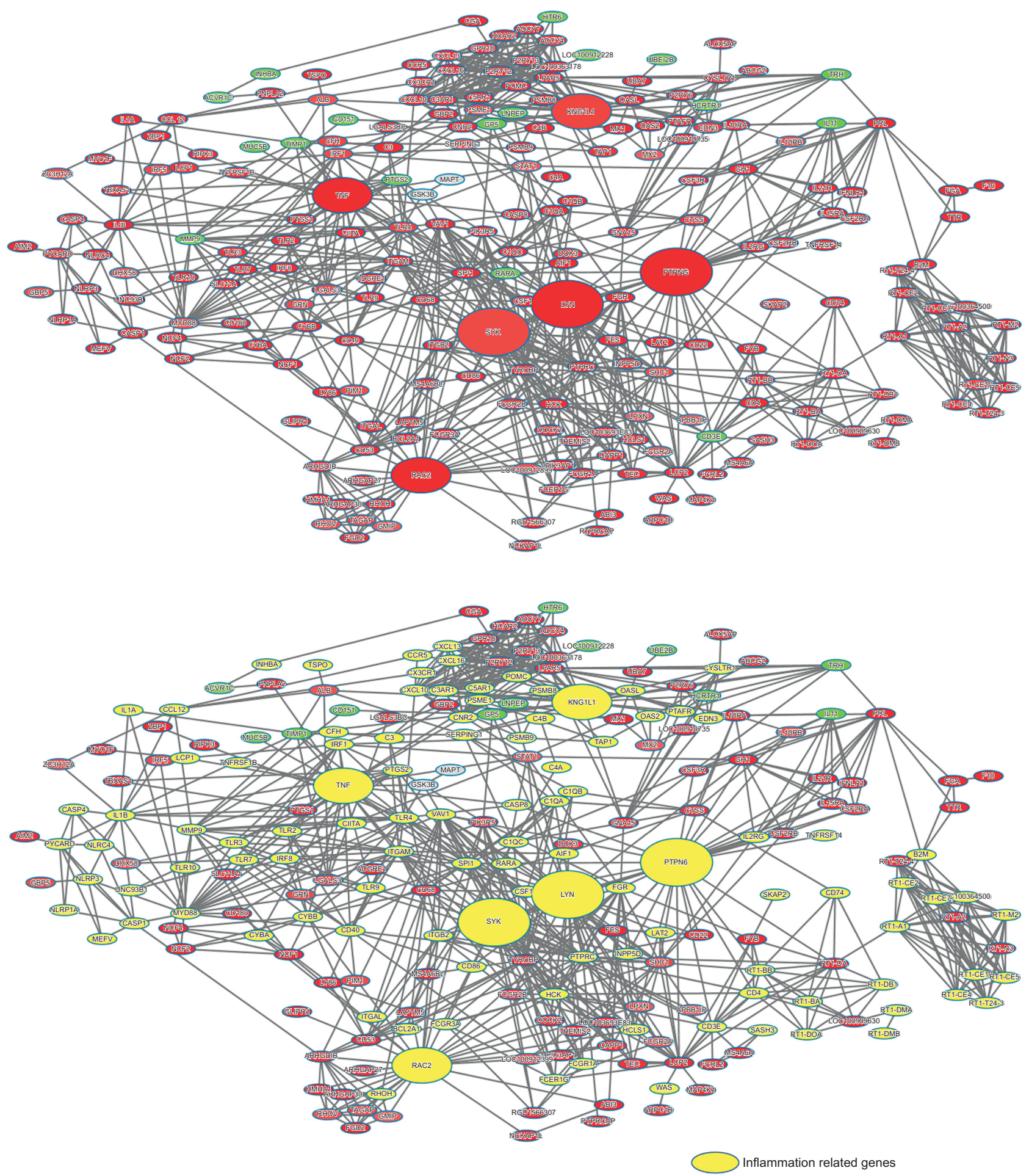

Fig. 2. Protein-protein interaction (PPI) network of genes differentially expressed by DAB in old rats. (A) Upregulated genes (red) and downregulated genes (green) on the PPI network, (B) Inflammation related genes (yellow) on the PPI network.

Previously we reported DAB causes tau hyperphosphorylation (Kang et al., 2017). In this study, we also found DABinduced an LPS-like pathway in old rats and altered TREM pathway-related inflammatory response in RNA-seq. TREM1 is an inflammation-related protein and its plasma concentra- tion is positively related with tau levels in Alzheimer's disease (Jiang et al., 2019).

We confirmed RNA-seq DEG results using RT-PCR. TREM-1 signaling via phosphorylation regulates IL-1 beta, NF-kB, TNF-alpha, and inflammatory cytokines (Colonna and 

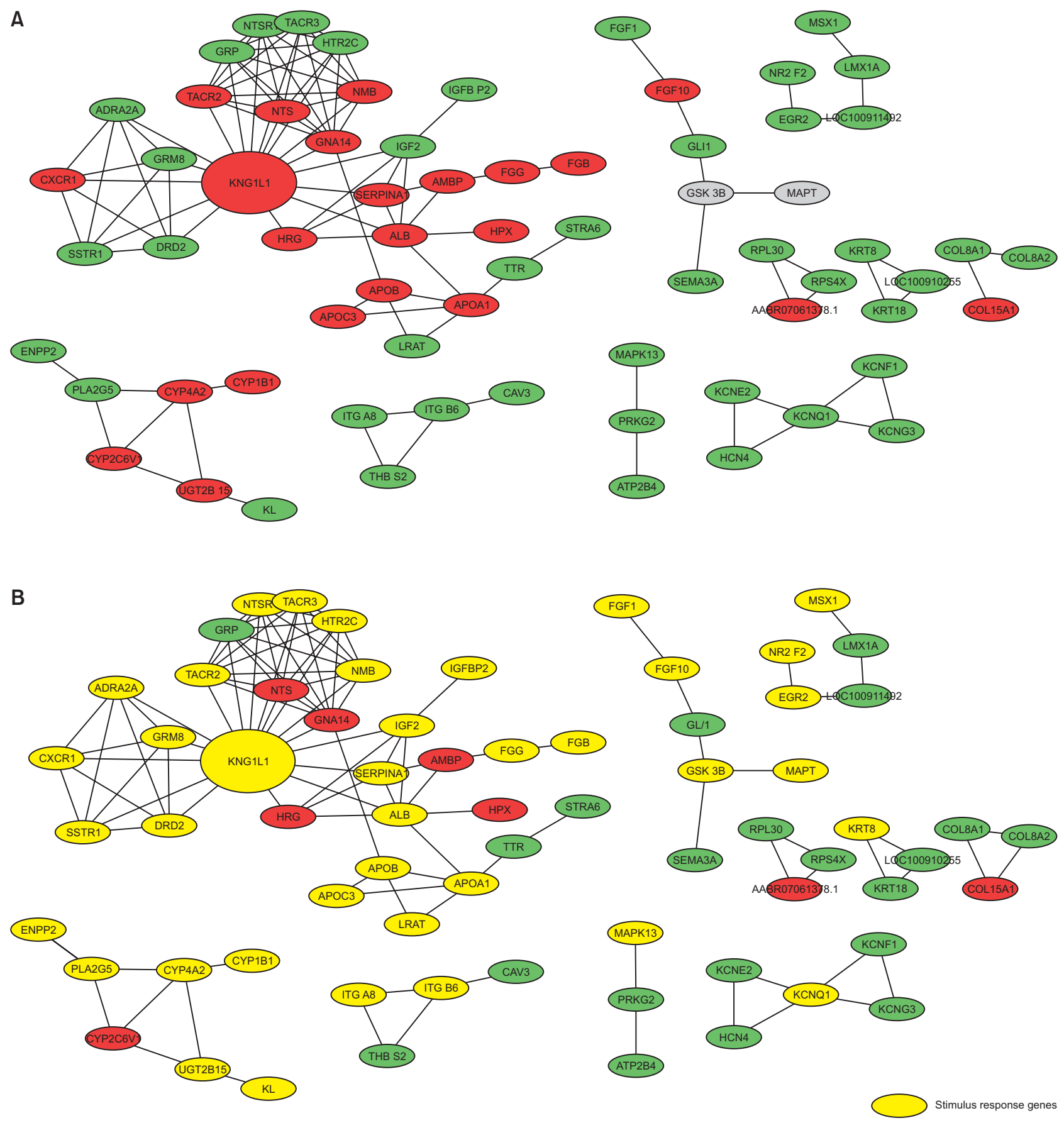

Fig. 3. PPI network of genes differentially expressed by DAB in young rats. (A) Upregulated genes (red) and downregulated genes (green) on the PPI network, (B) Stimulus response genes (yellow) on the PPI network.

Facchetti, 2003). Furthermore, we confirmed the TREM-1 pathway downstream protein, transmembrane adaptor protein DAP12 is an important contributor to inflammatory response (Tessarz and Cerwenka, 2008). In the present study, RT- PCR (Fig. 5) showed old rats exhibited greater gene expressional changes than young rats, and TREM1 was up-regulated by 3.5 and 1.3-fold in old and young rats, respectively. Some other TREM1-related genes, including TLR-4, C3a, C5a, DAP-12, Casp1, IL-1 $\beta$, and NF-kB, also showed higher fold changes in old rats. In young rats as well as smaller fold-changes RTPCR results were not in-line with RNA-seq results.

Toll-like receptors (TLRs) are a component of the innate immune system and recognize xenobiotics by acting as pattern recognition receptors. In brain, TLR-4 expression on the surfaces of microglia is a characteristic of neurodegenerative diseases such as AD, Multiple sclerosis, Parkinson's disease, amyotrophic lateral sclerosis (Fiebich et al., 2018). Microglia activation results in the inductions of inflammatory cytokines 


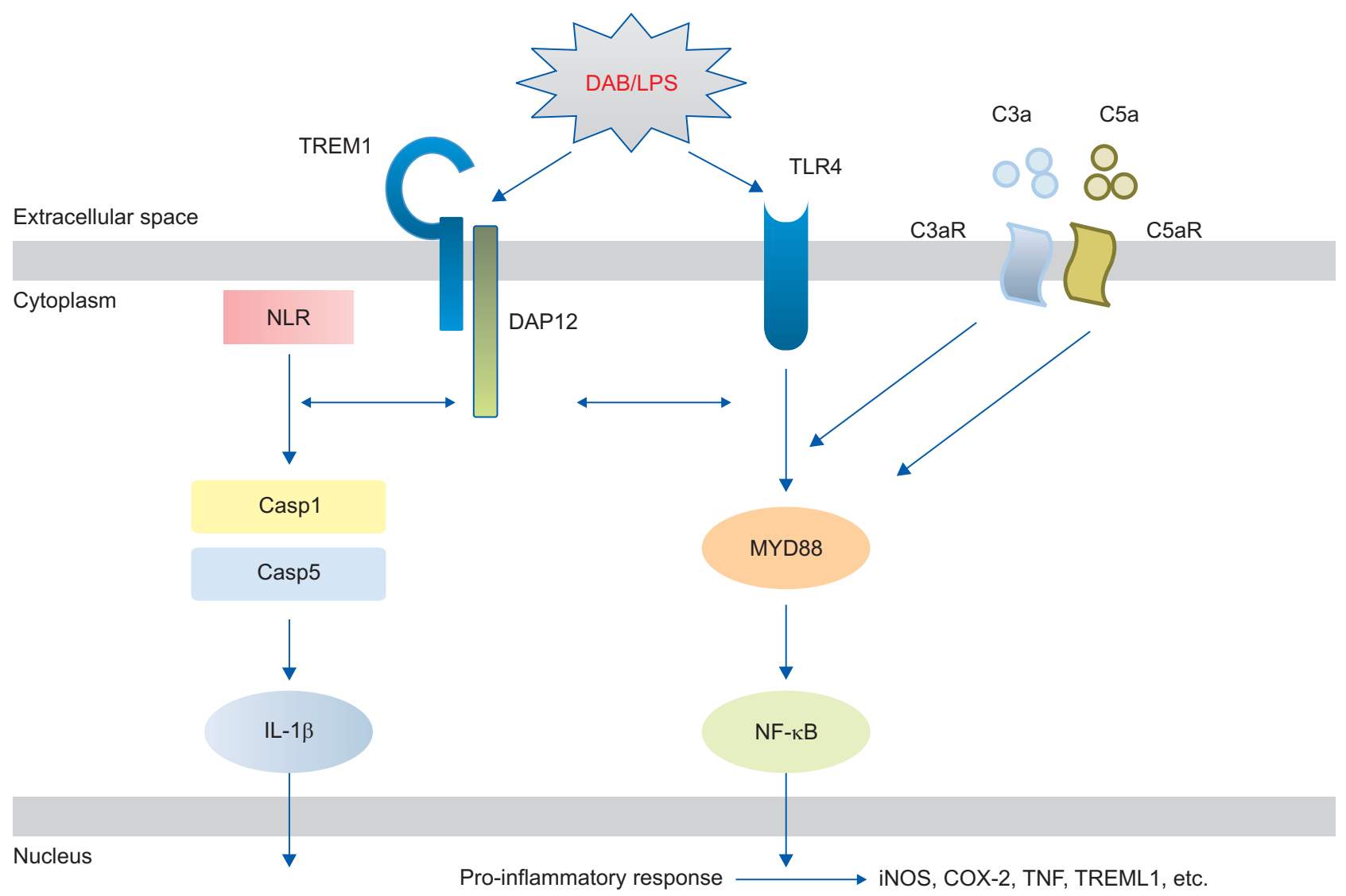

Fig. 4. Influences of DAB or LPS on the TREM1 signaling pathway. DAB/LPS activates TREM1 and initiates the classical pathway of complement activation. TREM1 interacts with DAP-12, activates inflammasome Casp1 and Casp5, and induces IL-1 $\beta$. The activations of TLR4 and complementary components (e.g., C3a and C5a) enhance MYD88 and NF-кB. Consequently, pro-inflammatory response induces the nuclear transcriptions of cytokines, chemokines, and cell surface molecules.

such as TNF-alpha, IL-1 beta, and IL-6, and these expressions are greater in the aged brain (Norden and Godbout, 2013), and we also observed TLR4 expression was elevated in old rats (Fig. 6A). Furthermore, it is known that TLR4 and MyD88-dependent signaling can stimulate the productions of NF-kB and inflammatory cytokines (Liu et al., 2017). Our study also found the upregulation of some other inflammation-related pathways such as TNF, SYK, and LYN. The role of these proteins was proven in the amyloidogenesis and tau phosphorylation of AD studies (Paris et al., 2014; Decourt et al., 2017; Gwon et al., 2019). Besides, KNG1L1 (kininogen 1-like 1 ), which is a protein related to the kallikrein-kinin system that participates in the inflammatory response and cytokines secretion, was found up-regulated in both young and old rats. Therefore, DAB partially induces its neurotoxicity via inflammation, but more strongly in old condition.

Amyloid precursor protein (APP), presenilin 1 (PSEN1), presenilin 2 (PSEN2), and apolipoprotein E (APOE) were reported as biomarkers of AD (Kim et al., 2014). The related proteins such as presenilin enhancer gamma-secretase subunit (PSENEN) and amyloid-beta (A4) precursor protein-binding (APBB1IP and APBA3, which interacts with APP), were upregulated by $D A B$ in old rats and suggested its association between $D A B$ and $A D$.

While xenobiotic metabolism-related proteins, cytochromes
P450 (CYP) - the most essential enzymes for phase I metabolism, were not changed by DAB in old rats, CYP- related proteins such as CYP4, CYP1, and UGT2 were upregulated by $\mathrm{DAB}$ treatment in young rats. CYP enzymes are involved in the phase I (modification) in the metabolism of xenobiotics, and UGT enzymes have the function in phase II (conjugation) of xenobiotic metabolites in which toxic compounds are conjugated with glucuronic acid. CYP and UGT proteins play important roles in the detoxification of toxicants in vivo (Rowland et al., 2013; Zanger and Schwab, 2013). Furthermore, the activities of CYP and UGT have been reported to be subject to age-related alterations (Vyskočilová et al., 2013). We also observed notable upregulations of other enzymes that also play important roles in drug metabolism such as flavine monooxygenases 9 (FMO9) and glutathione S-transferases alpha 5 (GSTA5) (FCs=99.5 and 789.3 respectively).

Aging causes physiologically significant changes to counteract xenobiotics. A significant reduction in liver volume reduces metabolic rates and the activities of some CYPs, whereas during detoxification, reduced kidney and liver blood flow diminish the excretion and elimination of xenobiotics and their metabolites (Klotz, 2009). Therefore, it appears a decline in the capacity to deal with xenobiotics during aging may underlie age-dependent sensitivity to DAB. We propose that instead of using metabolism and detoxification to reduce toxicant from 

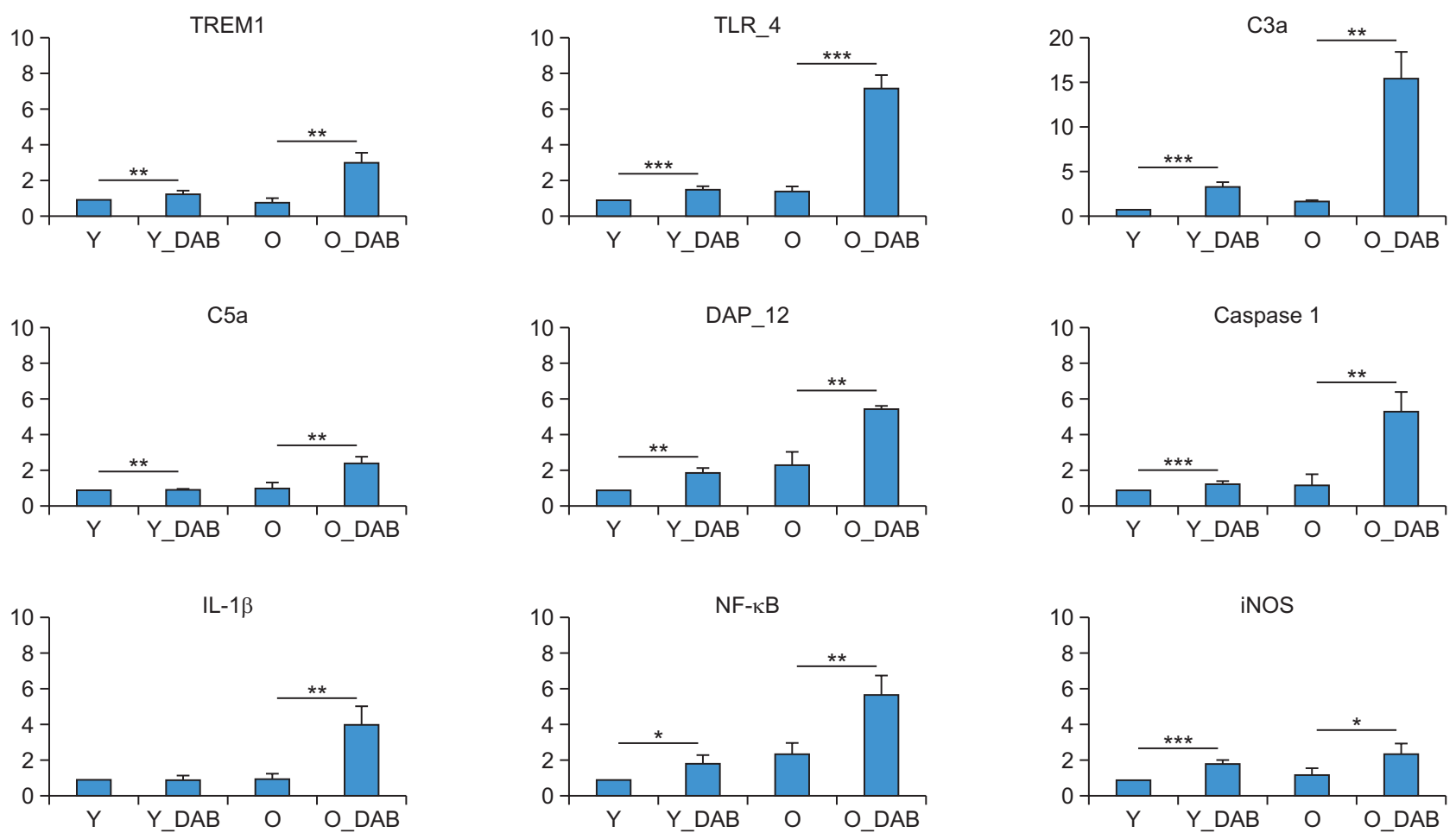

Fig. 5. RT-PCR results for DAB-induced expressional changes in the hippocampal tissues of young $(\mathrm{Y})$ and old $(\mathrm{O})$ rats. Fold changes in gene expression are expressed relative to non-treated young controls. Gene expressions were significantly more altered in old rats. ${ }^{*} p<0.05,{ }^{* *} p<0.01,{ }^{* * *} p<0.001$.

the body, old rats may respond to DAB via inflammation as the biological function declines by aging. Mild inflammation state by aging may contribute to DAB-induced toxicity in age rats (Bektas et al., 2018).

Notably, the prolactin (a hormone secreted by the pituitary gland) gene was upregulated more than any other gene ( $F C=78541.55$ ) by DEG in old rats but was downregulated in young rats. We also found that the gene expressions of prolactin receptors and dopamine receptors, which are involved in prolactin inhibition, varied in both young and old rats, e.g., $\mathrm{FC}$ of prolactin receptor in old and young rats were 1.93 and -95.3 , respectively. It is known that prolactin plays an important role in reproductive functions, and also acts as a cytokine, which is involved in immune response (Fojtíková et al., 2010). Therefore, we suggest additional studies be undertaken to determine the effect of DAB on the production of prolactin by age and gender.

Although our study reached the target to show the agedependent response to $D A B$ in young and old rats' hippocampus, there were still some limitations. Firstly, the hippocampus samples were divided into two parts for RNA-seq and RT-PCR, then the RNA samples of three rats per group were pooled to carry out RNA-seq while RT-PCR was performed on independent samples. However, the results of RT-PCR were consistent with RNA-seq in old rats. Secondly, our study only showed genomic data by using RNA-seq and RT-PCR. More studies are needed to explore the underlying mechanism of age-dependent responses to DAB.

Summarizing, we evaluated different responses to $D A B$ in young and old rats by RNA-seq. TREM1 signaling upregu-
A

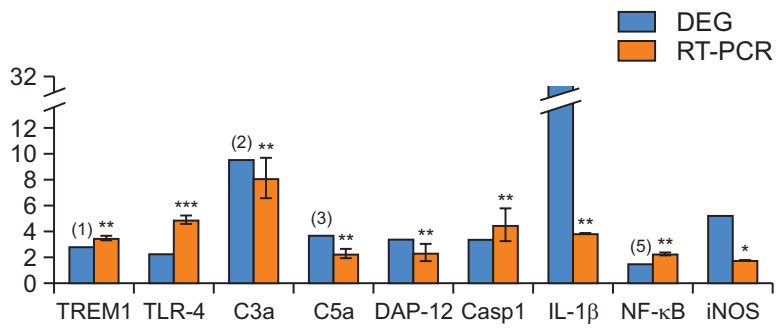

B

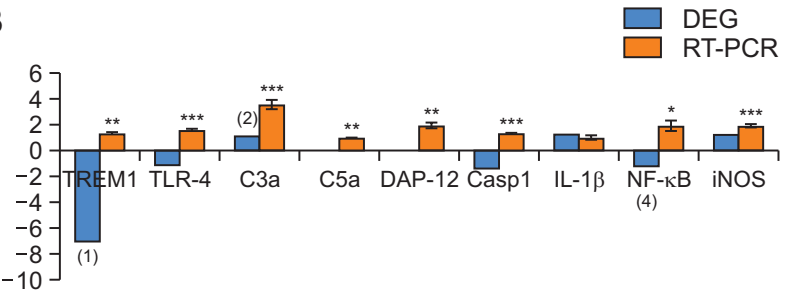

Fig. 6. Comparison of relative fold changes of genes involved in the TREM pathway as determined by RNA-seq and RT-PCR in old rats (A) and young rats (B). (1): TREML1. (2): C3. (3): average FC of C5aR1 and C5aR2. (4): NF-kB2. (5): average FC of NF-kB1 and NFkB2. We confirmed 9 TREM1 signaling-related genes using real-time PCR and found that fold-changes in gene expression as determined by RNA-seq, were consistent with real-time PCR results in old rats. However, there are some differences between RNA-seq and RT- PCR results were observed in young rats. ${ }^{*} p<0.05,{ }^{* *} p<0.01,{ }^{* * *} p<0.001$. 
lation, which is involved in $A D$, was confirmed in old rats by RT-PCR, but young rats showed inconsistent results. DAB increased inflammation-related pathways in old rats but enhanced metabolism and detoxification pathways in young rats. The above-mentioned findings indicate the toxicities of the neurotoxic organic solvents $D A B$ and $A D$ involve inflammatory response, and that this response is age dependent. The risk factors we found in this study are related to both $A D$ and other neurodegenerative diseases. It is crucial to develop a prevention strategy targeting the elderly population and workers at high-risk facilities to thwart or slow down disease progression, postpone AD onset, and reduce prevalence. Furthermore, establishing a feasible neurodegenerative disease management system should be a government priority, in which all people exposed to organic solvents should be monitored and controlled their risk factors yearly. We believe these strategies would effectively reduce the prevalence of neurogenerative diseases.

\section{CONFLICT OF INTEREST}

The authors declare no conflict of interests.

\section{ACKNOWLEDGMENTS}

This work was supported by a National Research Foundation of Korea (NRF) grant from the Korean government (MEST) (grant nos. NRF2013R1A1A3008851 and 2018R1D1A1B07049610).

\section{REFERENCES}

Anand, K. S. and Dhikav, V. (2012) Hippocampus in health and disease: an overview. Ann. Indian. Acad. Neurol. 15, 239-246.

Bektas, A., Schurman, S. H., Sen, R. and Ferrucci, L. (2018) Aging, inflammation and the environment. Exp. Gerontol. 105, 10-18.

Berr, C., Vercambre, M. N., Bonenfant, S., Manoux, A. S., Zins, M. and Goldberg, M. (2010) Occupational exposure to solvents and cognitive performance in the GAZEL cohort: preliminary results. Dement. Geriatr. Cogn. Disord. 30, 12-19.

Cavender, F. (1994) Aromatic hydrocarbons. In Patty's Industrial Hygiene And Toxicology (D. Clayton and F. Clayton, Eds.), pp. 1301. Wiley, New York.

Colonna, M. and Facchetti, F. (2003) TREM-1 (triggering receptor expressed on myeloid cells): a new player in acute inflammatory responses. J. Infect. Dis. 187 Suppl 2, S397-S401.

Costet, N., Béranger, R., Garlantézec, R., Rouget, F., Monfort, C., Cordier, S., Pelé, F. and Chevrier, C. (2018) Occupational exposure to organic solvents during pregnancy and childhood behavior: findings from the PELAGIE birth cohort (France, 2002-2013). Environ. Health 17, 63.

Decourt, B., Lahiri, D. K. and Sabbagh, M. N. (2017) Targeting tumor necrosis factor alpha for Alzheimer's disease. Curr. Alzheimer Res. 14, 412-425.

Denic, A., Glassock, R. J. and Rule, A. D. (2016) Structural and functional changes with the aging kidney. Adv. Chronic Kidney Dis. 23, 19-28.

Dick, F. D. (2006) Solvent neurotoxicity. Occup. Environ. Med. 63, 221$6,179$.

Dresselhaus, E. C. and Meffert, M. K. (2019) Cellular specificity of NF$\kappa B$ function in the nervous system. Front. Immunol. 10, 1043.

Dutta, G., Zhang, P. and Liu, B. (2008) The lipopolysaccharide Parkinson's disease animal model: mechanistic studies and drug discovery. Fundam. Clin. Pharmacol. 22, 453-464.

Fiebich, B. L., Batista, C. R. A., Saliba, S. W., Yousif, N. M. and de
Oliveira, A. C. P. (2018) Role of microglia TLRs in neurodegeneration. Front. Cell. Neurosci. 12, 329.

Fojtíková, M., Cerná, M. and Pavelka, K. (2010) A review of the effects of prolactin hormone and cytokine on the development and pathogenesis of autoimmune diseases. Vnitr. Lek. 56, 402-413.

Franke, H. A. (2014) Toxic stress: effects, prevention and treatment. Children (Basel) 1, 390-402.

Geller, A. M. and Zenick, H. (2005) Aging and the environment: a research framework. Environ. Health Perspect. 113, 1257-1262.

Gwon, Y., Kim, S. H., Kim, H. T., Kam, T. I., Park, J., Lim, B., Cha, H., Chang, H. J., Hong, Y. R. and Jung, Y. K. (2019) Amelioration of amyloid $\beta$-FcyRllb neurotoxicity and tau pathologies by targeting LYN. FASEB J. 33, 4300-4313.

Ji, F. and Sadreyev, R. I. (2018) RNA-seq: basic bioinformatics analysis. Curr. Protoc. Mol. Biol. 124, e68.

Jiang, T., Gong, P. Y., Tan, M. S., Xue, X., Huang, S., Zhou, J. S., Tan, L. and Zhang, Y. D. (2019) Soluble TREM1 concentrations are increased and positively correlated with total tau levels in the plasma of patients with Alzheimer's disease. Aging Clin. Exp. Res. 31, 1801-1805.

Kang, S. W., Kim, S. J. and Kim, M. S. (2017) Oxidative stress with tau hyperphosphorylation in memory impaired 1,2-diacetylbenzenetreated mice. Toxicol. Lett. 279, 53-59.

Kim, D. H., Yeo, S. H., Park, J. M., Choi, J. Y., Lee, T. H., Park, S. Y., Ock, M. S., Eo, J., Kim, H. S. and Cha, H. J. (2014) Genetic markers for diagnosis and pathogenesis of Alzheimer's disease. Gene 545, 185-193.

Kim, I. H., Kisseleva, T. and Brenner, D. A. (2015) Aging and liver disease. Curr. Opin. Gastroenterol. 31, 184-191.

Kim, M. K., Kim, K. S., Chung, J. H., Kim, J. H., Kim, J. R., Chung, H. Y. and Kim, M. S. (2007) Environmental metabolite, 1,2-diacetylbenzene, produces cytotoxicity through ROS generation in HUVEC cells. J. Toxicol. Environ. Health A 70, 1336-1343.

Kim, M. S., Park, H. R., Chung, H. Y., Kim, H. S., Yu, B. P., Yang, H. S. and Lee, J. (2011) Organic solvent metabolite, 1,2-diacetylbenzene, impairs neural progenitor cells and hippocampal neurogenesis. Chem. Biol. Interact. 194, 139-147.

Klotz, U. (2009) Pharmacokinetics and drug metabolism in the elderly. Drug Metab. Rev. 41, 67-76.

Kohl, M., Wiese, S. and Warscheid, B. (2011) Cytoscape: software for visualization and analysis of biological networks. Methods Mol. Biol. 696, 291-303.

Langmead, B. and Salzberg, S. L. (2012) Fast gapped-read alignment with Bowtie 2. Nat. Methods 9, 357-359.

Leng, N., Dawson, J. A., Thomson, J. A., Ruotti, V., Rissman, A. I., Smits, B. M., Haag, J. D., Gould, M. N., Stewart, R. M. and Kendziorski, C. (2013) EBSeq: an empirical Bayes hierarchical model for inference in RNA-seq experiments. Bioinformatics 29, 1035-1043.

$\mathrm{Li}, \mathrm{B}$. and Dewey, C. N. (2011) RSEM: accurate transcript quantification from RNA-Seq data with or without a reference genome. BMC Bioinformatics 12, 323.

Liguori, I., Russo, G., Curcio, F., Bulli, G., Aran, L., Della-Morte, D., Gargiulo, G., Testa, G., Cacciatore, F., Bonaduce, D. and Abete, P. (2018) Oxidative stress, aging, and diseases. Clin. Interv. Aging 13, 757-772.

Liu, T., Zhang, L., Joo, D. and Sun, S. C. (2017) NF-kB signaling in inflammation. Signal Transduct. Target. Ther. 2, 17023.

Norden, D. M. and Godbout, J. P. (2013) Review: microglia of the aged brain: primed to be activated and resistant to regulation. Neuropathol. Appl. Neurobiol. 39, 19-34.

Oliveira, A. R., Campos Neto, A. A., de Andrade, M. J. O., de Medeiros, P. C. B. and Dos Santos, N. A. (2018) Organic solvent exposure and contrast sensitivity: comparing men and women. Braz. J. Med. Biol. Res. 51, e6568.

Paris, D., Ait-Ghezala, G., Bachmeier, C., Laco, G., Beaulieu-Abdelahad, D., Lin, Y., Jin, C., Crawford, F. and Mullan, M. (2014) The spleen tyrosine kinase (Syk) regulates Alzheimer amyloid- $\beta$ production and Tau hyperphosphorylation. J. Biol. Chem. 289, 3392733944.

Rowland, A., Miners, J. O. and Mackenzie, P. I. (2013) The UDP-glucuronosyltransferases: their role in drug metabolism and detoxification. Int. J. Biochem. Cell Biol. 45, 1121-1132. 
Sabri, M. I., Hashemi, S. B., Lasarev, M. R. and Spencer, P. S. (2007) Axonopathy-inducing 1,2-diacetylbenzene forms adducts with motor and cytoskeletal proteins required for axonal transport. Neurochem. Res. 32, 2152-2159.

Sarkar, S. N., Russell, A. E., Engler-Chiurazzi, E. B., Porter, K. N. and Simpkins, J. W. (2019) MicroRNAs and the genetic nexus of brain aging, neuroinflammation, neurodegeneration, and brain trauma. Aging Dis. 10, 329-352.

Sengupta, P. (2013) The laboratory rat: relating its age with human's. Int. J. Prev. Med. 4, 624-630.

Stambler, I., Jin, K., Lederman, S., Barzilai, N., Olshansky, S. J., Omokaro, E., Barratt, J., Anisimov, V. N., Rattan, S., Yang, S., Forster, M. and Byles, J. (2018) Aging health and R\&D for healthy longevity must be included into the WHO work program. Aging Dis. 9, 331333.

Szklarczyk, D., Franceschini, A., Wyder, S., Forslund, K., Heller, D., Huerta-Cepas, J., Simonovic, M., Roth, A., Santos, A., Tsafou, K. P., Kuhn, M., Bork, P., Jensen, L. J. and von Mering, C. (2015) STRING v10: protein-protein interaction networks, integrated over the tree of life. Nucleic Acids Res. 43, D447-D452.

Tessarz, A. S. and Cerwenka, A. (2008) The TREM-1/DAP12 pathway. Immunol. Lett. 116, 111-116.

Thrall, K. D., Gies, R. A., Cartmell, A. M., Wu, H., Soelberg, J. J. and Klein, J. A. (2007) A liquid chromatographic-mass spectrometric method to evaluate the distribution kinetics of 1,2-diethylbenzene and its metabolite 1,2-diacetylbenzene in the F344 male rat. $J$. Toxicol. Environ. Health A 70, 67-72.

Vyskočilová, E., Szotáková, B., Skálová, L., Bártíková, H., Hlaváčová, J. and Boušová, I. (2013) Age-related changes in hepatic activity and expression of detoxification enzymes in male rats. Biomed. Res. Int. 2013, 408573.

Wood, R. L. and Liossi, C. (2005) Long-term neuropsychological impact of brief occupational exposure to organic solvents. Arch. Clin. Neuropsychol. 20, 655-665.

Zanger, U. M. and Schwab, M. (2013) Cytochrome P450 enzymes in drug metabolism: regulation of gene expression, enzyme activities, and impact of genetic variation. Pharmacol. Ther. 138, 103-141. 